\title{
Métodos de ajuste de semivariogramas para modelagem espacial de íons de precipitação pluvial em Cuiabá, Brasil
}

\author{
Methods of variogram fitting for the rainfall ion spatial modeling in Cuiabá, Brazil
}

\author{
Vanessa Rakel de Moraes Dias ${ }^{* 1}$, Marcelo de Carvalho Alves² ${ }^{2}$ Luciana Sanches $^{3}$ \\ ${ }^{1}$ Programa de Pós-Graduação em Física Ambiental - Universidade Federal de Mato Grosso, Brasil. \\ ${ }^{2}$ Departamento de Solos e Engenharia Rural - Universidade Federal de Mato Grosso, Brasil. \\ ${ }^{3}$ Departamento de Engenharia Sanitária e Ambiental, Programa de Pós-Graduação em Física Ambiental - Universidade Federal \\ de Mato Grosso, Brasil.
}

\begin{abstract}
Resumo
O mapeamento de variáveis químicas da precipitação é de fundamental importância para o conhecimento e compreensão de fontes de emissão de poluentes atmosféricos. Para isso, métodos de interpolação espacial devem ser avaliados para subsidiar o mapeamento destas variáveis químicas. Assim, o objetivo deste trabalho foi avaliar métodos de modelagem de semivariograma que melhor se ajustaram às variáveis $\mathrm{pH}$, condutividade elétrica, nitrato e sulfato da precipitação em Cuiabá, Mato Grosso. Foram avaliados os métodos de ajuste dos mínimos quadrados ordinários (OLS-Ordinaty Least Squares) e da máxima verossimilhança restrita (REML-Restricted Maximum Likelihood). Os critérios de seleção do melhor método foram o critério de informação de Akaike (AIC) e o desvio padrão dos erros reduzidos (SER) gerados pela validação cruzada. O método da máxima verossimilhança restrita foi o mais adequado para o mapeamento das variáveis químicas da precipitação em Cuiabá. A análise da estrutura dos semivariogramas foi importante porque indicou fontes de emissão local (urbana) e regional (queimadas) de partículas de aerossóis e gases, considerando a redução do alcance das variáveis analisadas de outubro a fevereiro.
\end{abstract}

Palavras-chave: Geoestatística. Chuva. Modelagem espacial. Nitrato. Sulfato

\begin{abstract}
The mapping of ion precipitation is of fundamental importance to knowledge and understanding of emission sources of air pollutants. Therefore, spatial interpolation methods should be assessed for support the mapping of these ions. In this context, the aim of this study was to assess the semivariogram modeling methods that best fit the variables $p H$, electrical conductivity, nitrate and sulfate precipitation in Cuiabá, Mato Grosso, Brazil. We assessed the methods ordinary least squares (OLS) and residual maximum likelihood (REML). The criteria for selecting the best method were the Akaike information criterion (AIC) and the standard deviation of the reduced errors (SER) generated by cross-validation. The restricted maximum likelihood method was the best for mapping ion precipitation in Cuiabá. Analysis of the structure of the semivariogram was important because it indicated local emission sources (urban) and regional (burning) of aerosol particles and gases.
\end{abstract}

Keywords: Geostatistics. Rainfall. Spatial modeling. Nitrate. Sulfate 


\section{Introdução}

$\mathrm{O}$ aumento das emissões de poluentes atmosféricos, principalmente dióxido de enxofre $\left(\mathrm{SO}_{2}\right)$, óxidos de nitrogênio $\left(\mathrm{NO}_{\mathrm{x}}\right)$, compostos orgânicos voláteis (VOCs) e aerossóis, está relacionado ao crescimento da população urbana que demanda maior produção de energia por meio da queima de combustíveis fósseis, sendo esta uma das principais fontes de emissão desses poluentes atmosféricos (MIGLIAVACCA et al., 2005).

Além da queima de combustíveis (gás natural, carvão, gasolina, álcool, diesel) e processos industriais (fundições, refinarias, fabricação de fertilizantes, papel, entre outras) destacam-se as queimadas em florestas ou pastagens e a suspensão de material particulado do solo (FORNARO, 2006). As queimadas são as principais fontes antropogênicas de emissão de gases e partículas de aerossóis durante o período seco nas regiões Centro-Oeste e Norte do Brasil, alterando a química da atmosfera (ANDREAE et al., 1994; ANDREAE et al., 2001), causando danos aos ecossistemas e alterando a qualidade do ar e, consequentemente, a qualidade de vida da população.

Poluentes como, óxidos de enxofre e nitrogênio, sais, metais e orgânicos, entre outros, emitidos para a atmosfera a partir de fontes naturais e antropogênicas, quando não sofrem deposição seca, são adsorvidos pelas partículas em suspensão ou em suas formas de vapor, e são carregados por precipitação quando entram em contato com gotas de chuva (SINGH et al., 2007).

As deposições atmosféricas constituem um dos principais mecanismos da ciclagem e redistribuição dos vários elementos químicos sobre a superfície terrestre, exercendo, portanto, um papel fundamental nos processos biogeoquímicos continentais e costeiros. Essas deposições podem resultar em impacto negativo nos mananciais, na biota e sobre os materiais expostos a essa deposição (LEAL et al., 2004; SOUZA et al., 2006). Somado a esses impactos negativos estão os problemas relacionados aos efeitos adversos à saúde humana que pode estar exposta de maneira direta, por inalação de material particulado suspenso no ar, ou indireta, por ingestão de água ou alimentos contaminados, ou mesmo pela adsorção pela pele (CARMO et al., 2010).

Assim, é relevante o conhecimento quantitativo e qualitativo dessas deposições para o entendimento da influência das atividades antropogênicas nestes processos. Neste contexto, é importante conhecer a estrutura e a magnitude de variáveis químicas da precipitação em qualquer local no espaço, o que é normalmente impossível, tanto financeira quanto operacionalmente, tornando-se importante o uso de métodos de interpolação como o geoestatístico que estima valores em locais não amostrados, a partir de dados vizinhos coletados em malha ao longo do espaço e do tempo (LOPHAVEN et al., 2004).
A geoestatística envolve análises e predições de fenômenos regionalizados (CRESSIE, 1993), tais como: nutrientes do solo, precipitação pluviométrica, concentração de poluentes em ambientes aquáticos, entre outros. Elementos atmosféricos têm variações temporais e espaciais (AL-KHASHMAN e TARAWNEH, 2007), sendo que a análise temporal da composição química da precipitação tem sido amplamente explorada (FLUES et al., 2002; ROCHA et al., 2003; ANDRÉ et al., 2007; PAULIQUEVIS et al., 2007; RODRIGUES et al., 2007; TEIXEIRA et al, 2008; FONTENELE et al., 2009; HAN et al., 2010). Porém, a variabilidade espacial tem sido ignorada nos estudos desenvolvidos no Brasil, mas tem sido considerada na Europa e nos Estados Unidos, por possuírem uma rede de monitoramento consolidada.

Como a composição química da precipitação é uma variável regionalizada, devido à composição iônica que varia no espaço por causa das diferenças das fontes locais, sua variabilidade espacial pode ser analisada por técnicas geoestatísticas (CAO et al., 2009). Os primeiros estudos de variabilidade espacial da composição química da precipitação foram desenvolvidos por Cressie (1993) nos Estados Unidos, com dados de 19 estações da UAPSP (Utility Acid Precipitation Study Program) coletados entre 1982 e 1983, para avaliar a distribuição da acidez da precipitação ao longo do espaço.

Dentro deste contexto, o objetivo deste trabalho foi estudar a estrutura de dependência espacial das variáveis $\mathrm{pH}$, condutividade elétrica, nitrato e sulfato da precipitação pluvial em Cuiabá, Mato Grosso, avaliando-se os métodos de ajuste de semivariograma teórico por mínimos quadrados ordinários (OLS - Ordinaty Least Squares) e máxima verossimilhança restrita (REML Restricted Maximum Likelihood).

\section{Material e Métodos}

\subsection{Descrição da área de estudo}

O estudo foi desenvolvido em Cuiabá, capital do Estado de Mato Grosso, que possui uma área de 3.538,167 $\mathrm{Km}^{2}$, localizada na região Centro-Oeste do Brasil. A cidade situa-se no centro geodésico da América do Sul, nas coordenadas $15^{\circ} 35^{\prime} 56^{\prime \prime}$ latitude Sul e $56^{\circ} 06^{\prime} 01^{\prime \prime}$ longitude Oeste.

Devido à expansão urbana, ocorreu a conurbação entre a cidade de Cuiabá e Várzea Grande, formando o Aglomerado Urbano Cuiabá-Várzea Grande, com população em torno de 800 mil habitantes, sendo $552 \mathrm{mil}$ de Cuiabá (BRASIL, 2012), correspondendo ao grande centro comercial e de serviços do Estado. Cuiabá possui indústrias de extração de minerais metálicos e não-metálicos, fabricação de produtos alimentícios, bebidas, produtos têxteis, produtos de madeira, celulose e papel, preparação de couros, fabricação de produtos derivados de biocombustíveis, além de confecções em geral (MATO 
GROSSO, 2009), porém, são na maioria de pequeno ou médio porte. Na região, há uma frota de aproximadamente 637 mil veículos automotores estimados em 2012 (BRASIL, 2012).

O clima da região é considerado tropical semi-úmido, tipo Aw segundo a classificação de Köppen, com um período chuvoso entre os meses de outubro a abril e outro seco de maio a setembro, com índice médio de precipitação anual de $1.500 \mathrm{~mm}$ e a temperatura média mensal varia entre $21,9^{\circ} \mathrm{C}$ e $31,3^{\circ} \mathrm{C}$ (MAITELLI, 1994).

\subsection{Amostragem da precipitação}

Foram selecionados 26 locais para amostragem de precipitação abrangendo a área urbana de Cuiabá com alguns pontos de coleta no seu entorno, inclusive nas cidades de Várzea Grande, Santo Antônio do Leverger e no Distrito de Nossa Senhora da Guia, compreendendo uma área de estudo de aproximadamente $730.000 \mathrm{~m}^{2}$ (Figura 1). As coordenadas geográficas dos locais selecionados foram obtidas por um GPS (Garmin, modelo 76 CSX, USA).

As amostras de precipitação foram coletadas em 20/10/2009, 07/12/2009 e 10/02/2010, utilizando-se coletores manuais de polietileno encaixados em suportes de metal a 2 metros de altura. Além de $\mathrm{pH}$ e condutividade elétrica, foram analisados os ânions nitrato $\left(\mathrm{NO}_{3}^{-}\right) \mathrm{e}$ sulfato $\left(\mathrm{SO}_{4}^{2-}\right)$, utilizando-se um cromatógrafo iônico (Dionex, modelo ICS 90, USA).

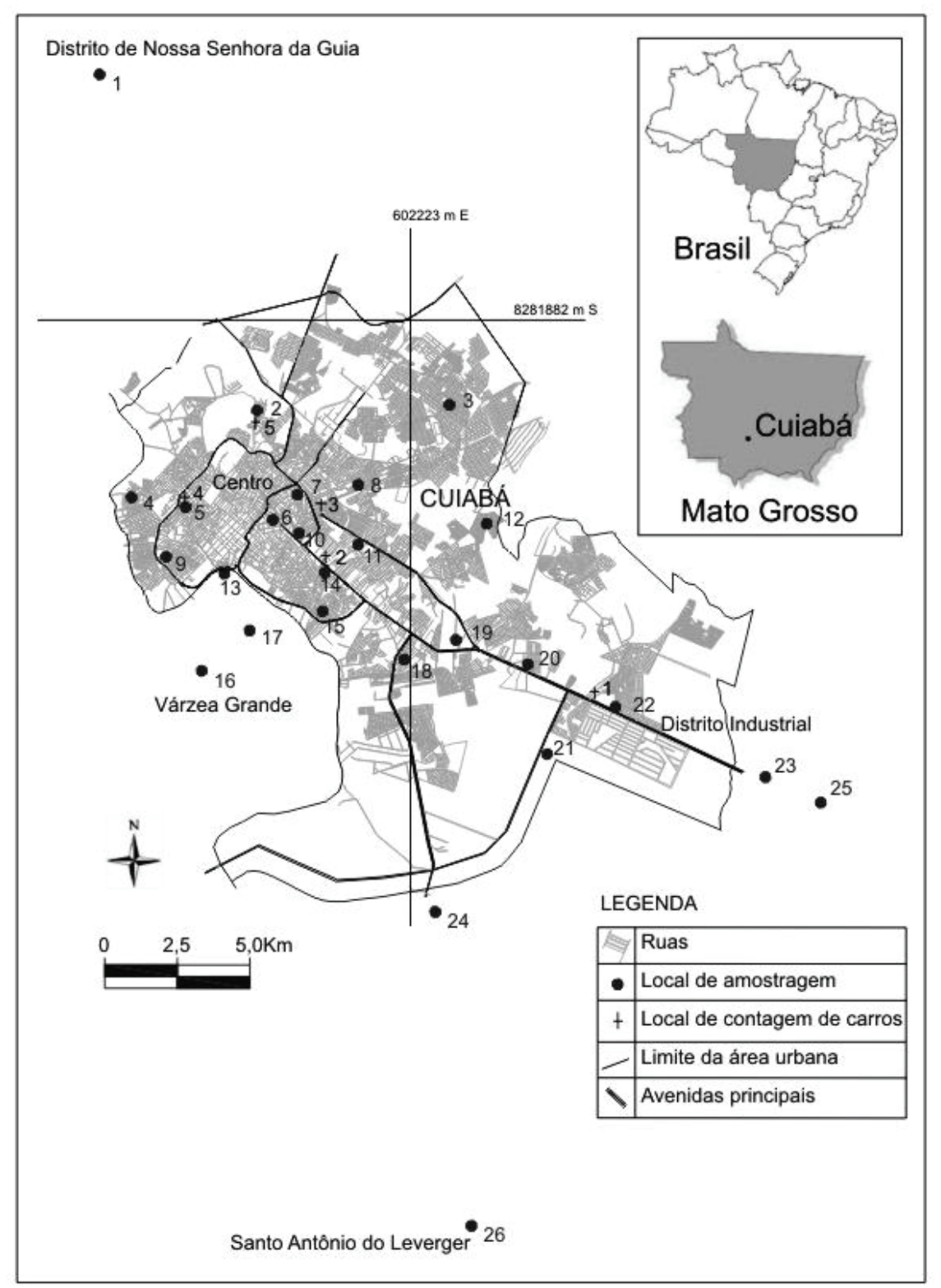

Figura 1 - Mapa da localização da área urbana de Cuiabá e locais de amostragem de precipitação pluvial 


\subsection{Geoestatística}

Em Geoestatística, é importante a seleção do melhor método de ajuste de semivariograma porque, a partir deste, interpreta-se a estrutura de correlação espacial a ser utilizada nos procedimentos inferenciais da krigagem. O modelo relaciona a semivariância com o vetor distância segundo a equação (1):

$$
\widehat{\gamma}(h)=\frac{1}{2 N(h)} \sum_{i=1}^{N(h)}\left[z\left(x_{i}\right)-z\left(x_{i}+h\right)\right]^{2}
$$

em $\hat{\gamma}(h)$ que é a semivariância estimada para cada distância (lag) entre pares de pontos, $N(h)$ é o número de pares de valores medidos $z(x i), z(x i+h)$, separados pela distância $h$ (BURROUGH e McDONNELL, 1998).

O modelo esférico foi utilizado no presente estudo por apresentar um alcance prático em a, ou seja, no alcance, em que o patamar é igual a $\mathrm{C}_{0}+\mathrm{C}$ (ANDRIOTTI, 2009). Enquanto outros modelos como, por exemplo, exponencial e gaussiano alcançam o patamar assintoticamente (CRESSIE, 1993), tendo um significado puramente analítico, em que o patamar é alcançado pela curva de forma assintórica, ou seja, teoricamente quando $h=\infty$.

O modelo esférico foi ajustado tanto pelo método dos mínimos quadrados ordinários, quanto pelo método de máxima verossimilhança restrita, segundo a equação (2):

$$
\gamma(h)=\left\{\begin{array}{c}
C_{0}+C_{1}\left(\frac{3}{2} \frac{h}{a}-\frac{1}{2}\left(\frac{h}{a}\right)^{3}\right), 0 \leq h<a \\
C_{0}+C_{1}, h \geq a
\end{array}\right.
$$

em que $C_{o}$ é o efeito pepita, $C_{0}+C_{1}$ o patamar, $a$ o alcance e $h$ a distância.

O método dos mínimos quadrados ordinários consiste em obter os valores dos parâmetros de um modelo que minimiza a soma do quadrado da diferença entre os valores observados e os estimados (MELLO et al., 2005). O método da máxima verossimilhança restrita corresponde ao ajuste de um modelo direto aos dados e consiste em maximizar a função de densidade de probabilidade em relação aos efeitos fixos e aos componentes de variância dos efeitos aleatórios do modelo, obtendo-se a partir de uma amostra o estimador "mais verossímil" dos parâmetros de certo modelo probabilístico (OLIVEIRA, 2003).

Para avaliar o desempenho dos dois métodos, mínimos quadrados ordinários (OLS - Ordinaty Least Squares) e máxima verossimilhança restrita (REML - Restricted Maximum Likelihood), foi utilizado o critério de informação de Akaike (AIC), segundo a equação (3):

$$
A I C=-2 \ln (L)+2 p
$$

em que $L$ é a verossimilhança maximizada do método candidato e $p$ o número de parâmetros desse método (RIBEIRO JR. e DIGGLE, 2001). Menores valores de AIC indicam o melhor método.

A técnica de validação cruzada foi utilizada como critério de comparação, que consiste em retirar temporariamente do conjunto de dados um valor observado e estimá-lo a partir dos outros, esse processo é feito para todos os valores observados. Posteriormente foi calculado o desvio padrão dos erros reduzidos $\left(S_{E R}\right)$ (CRESSIE, 1993), segundo a equação (4):

$$
S_{E R}=\sqrt{\frac{1}{n} \sum_{i=1}^{n}\left(\frac{z\left(x_{i 0}\right)-\bar{z}\left(x_{i 0}\right)}{\sigma_{i 0}}\right)^{2}}
$$

em que $z\left(x_{i 0}\right)$ é o valor observado no ponto i0, é o valor estimado para o ponto i0 e $\sigma\left(x_{i 0}\right)$ é o desvio padrão da krigagem no ponto i0. O desvio padrão dos erros reduzidos deve ser próximo de 1.

Foi utilizado o programa R (R Core Team, 2014. R: A language and environment for statistical computing. $\mathrm{R}$ Foundation for Statistical Computing, Vienna, Austria). Assim como o pacote de análise geoestatística geoR (RIBEIRO JR. e DIGGLE, 2001). O R está disponível como programa livre (http://www.r-project.org/).

Para as variáveis que apresentaram semelhança em relação ao parâmetro alcance, foram feitas análises de correlação sendo que, para testar se o coeficiente de correlação de Pearson (r) foi significativamente diferente de zero, ao nível de significância de $5 \%$, testou-se a hipótese nula $\mathrm{r}=0$, segundo BRAULE, 2001.

\section{Resultados e Discussão}

\subsection{Seleção do método de ajuste dos semiva- riogramas}

A estatística descritiva das variáveis químicas da precipitação foi reportada em Dias et al. (2012). Os conjuntos de dados foram posteriormente transformados utilizando-se logaritmo natural para se obter distribuições normais para os procedimentos geoestatísticos (RIBEIRO JR. e DIGGLE, 2001). Os dados de pH não foram transformados porque a função $\mathrm{pH}$ é definida como o logaritmo negativo da concentração hidrogeniônica [H+]. A distribuição log-normal das concentrações da precipitação foram reportadas por outros autores (PIO et al., 1991; DURAND et at., 1992; ALASTUEY et al., 1999), sendo que a log-transformação é comum para variáveis químicas da precipitação (TRANTER et al., 1987; GALPIN e HELD, 2002).

Para selecionar um método de ajuste variográfico 
não é suficiente apenas a análise do comportamento dos semivariogramas, pois essa é uma avaliação subjetiva. Assim, a utilização do critério de informação de Akaike (AIC) e técnicas de validação cruzada (desvio padrão do erro reduzido $\left(S_{E R}\right)$ ) são avaliações mais objetivas (Tabela 1). A indicação do melhor método de ajuste de acordo com os critérios adotados está em negrito.

Valores de AIC menores indicaram o melhor método a ser selecionado, assim como SER próximo de 1.

Comparando-se os valores de Critério de Informação de Akaike (AIC), e desvio padrão do erro reduzido (SER) entre os dois métodos, o método de máxima verossimi- lhança restrita apresentou superioridade em relação ao outro método.

Kerry e Oliver (2007) observaram superioridade do método de máxima verossimilhança em relação aos mínimos quadrados, quando compararam semivariogramas de propriedades do solo, e observaram que as previsões com base no primeiro método eram mais precisas do que os semivariogramas estimados pelo método dos mínimos quadrados para menos de 100 locais de amostragem.

Esse método é uma técnica de estimação usada com freqüência porque possui propriedades assintóticas interessantes: eficiência e consistência (MELLO et al., 2005).

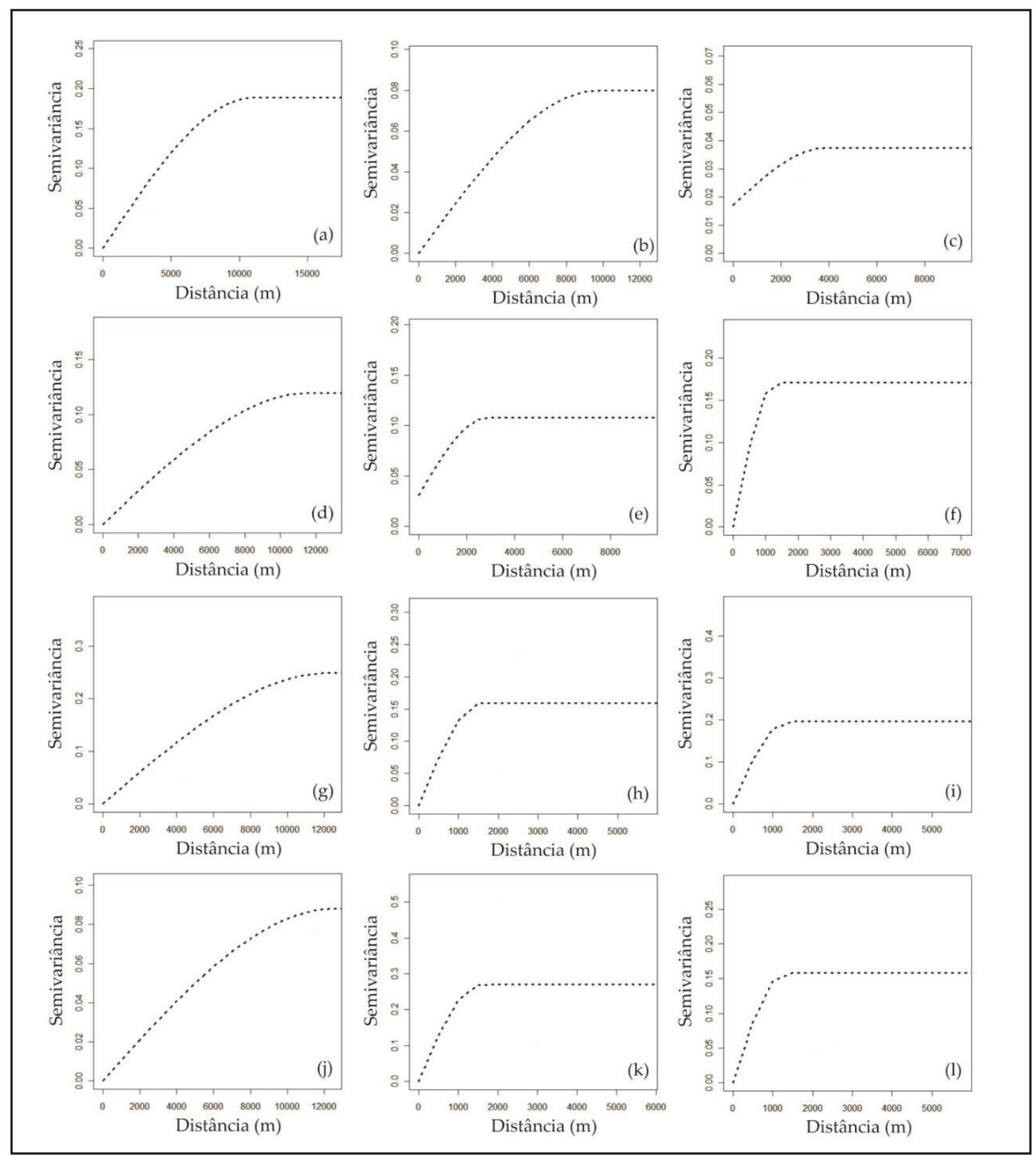

Figura 2 - Semivariogramas com modelo esférico ajustado pelo método REML das variáveis pH (a), (b) e (c), condutividade elétrica (d), (e) e (f), nitrato (g), (h) e (i), e sulfato (j), (k) e (l), em outubro, dezembro e fevereiro, respectivamente 
Tabela 1 - Critério de Informação de Akaike (AIC) e desvio padrão do erro reduzido ( $\left.S_{E R}\right)$ para o modelo esférico para os métodos dos mínimos quadrados ordinário (OLS) e máxima verossimilhança restrita (REML), para amostragens de outubro, dezembro e fevereiro

\begin{tabular}{llllll}
\hline \multirow{2}{*}{ Variável } & Mês & OLS & \multicolumn{3}{l}{ REML } \\
\cline { 3 - 6 } & out & AIC & $S_{\text {ER }}$ & $A I C$ & $S_{\text {ER }}$ \\
\hline $\mathrm{pH}$ & 27,03 & 1,50 & 19,97 & 1,12 \\
& dez & 1,24 & 0,96 & 0,91 & 1,00 \\
& fev & $-0,90$ & 1,31 & $-3,98$ & 1,01 \\
\hline $\mathrm{EC}$ & out & 108,80 & 1,91 & 100,40 & 1,42 \\
& dez & 152,89 & 2,34 & 122,60 & 1,11 \\
& fev & 122,38 & 1,21 & 119,60 & 1,16 \\
\hline $\mathrm{NO}_{3}{ }^{-}$ & out & 316,51 & 0,97 & 315,80 & 0,92 \\
& dez & 305,17 & 0,79 & 303,20 & 0,93 \\
& fev & 304,98 & 1,34 & 301,90 & 1,28 \\
\hline $\mathrm{SO}_{4}{ }^{2-}$ & out & 274,90 & 0,79 & 273,60 & 0,89 \\
& dez & 312,98 & 0,79 & 310,20 & 1,64 \\
& fev & 298,17 & 1,50 & 295,60 & 1,16 \\
\hline
\end{tabular}

\subsection{Parâmetros dos semivariogramas}

A dependência espacial das variáveis físico-químicas ( $\mathrm{pH}$ e condutividade elétrica) e químicas $\left(\mathrm{NO}_{3}^{-}\right.$e $\mathrm{SO}_{4}{ }^{2-}$ ) foi constatada por meio dos modelos variográficos. $\mathrm{O}$ modelo de semivariograma isotrópico esférico foi ajustado pelos métodos dos mínimos quadrados ordinários (OLS) e da máxima verossimilhança restrita (REML) para as três amostragens em: outubro, dezembro e fevereiro. Pelo ajuste dos semivariogramas foram obtidos os parâmetros: efeito pepita $\left(C_{0}\right)$, patamar $\left(C_{0}+C_{1}\right)$ e alcance (a) (Tabela 2).

O parâmetro alcance estimado foi maior em outubro do que dezembro e fevereiro, correspondendo a aproximadamente o raio da área urbana de Cuiabá em que abrangeu este estudo (com diâmetro maior de

Tabela 2 - Efeito pepita $\left(C_{0}\right)$, patamar $\left(C_{0}+C_{1}\right)$ e alcance (a) estimados para o modelo esférico segundo os métodos dos mínimos quadrados ordinários (OLS) e máxima verossimilhança restrita (REML), em outubro, dezembro e fevereiro

\begin{tabular}{|c|c|c|c|c|c|c|c|}
\hline \multirow{2}{*}{ Variável } & \multirow{2}{*}{ Mês } & \multicolumn{3}{|l|}{ OLS } & \multicolumn{3}{|c|}{ REML } \\
\hline & & $C_{o}$ & $C_{o}+C_{1}$ & $a$ & $C_{o}$ & $C_{o}+C_{1}$ & $a$ \\
\hline \multirow[t]{3}{*}{$\mathrm{pH}$} & out & 0,01 & 0,11 & 13862 & 0,00 & 0,19 & 11049 \\
\hline & dez & 0,00 & 0,08 & 9194 & 0,00 & 0,08 & 9668 \\
\hline & fev & 0,00 & 0,04 & 5785 & 0,02 & 0,02 & 3866 \\
\hline \multirow[t]{3}{*}{$\mathrm{EC}$} & out & 0,00 & 0,09 & 13050 & 0,00 & 0,12 & 11695 \\
\hline & dez & 0,00 & 0,13 & 9278 & 0,03 & 0,08 & 2868 \\
\hline & fev & 0,00 & 0,20 & 2360 & 0,00 & 0,17 & 1306 \\
\hline \multirow[t]{3}{*}{$\mathrm{NO}_{3}{ }^{-}$} & out & 0,00 & 0,23 & 13500 & 0,00 & 0,25 & 12318 \\
\hline & dez & 0,00 & 0,16 & 2975 & 0,00 & 0,16 & 1578 \\
\hline & fev & 0,01 & 0,18 & 2749 & 0,00 & 0,19 & 1362 \\
\hline \multirow[t]{3}{*}{$\mathrm{SO}_{4}{ }^{2-}$} & out & 0,00 & 0,11 & 12587 & 0,00 & 0,09 & 12544 \\
\hline & dez & 0,00 & 0,29 & 1534 & 0,00 & 0,27 & 1536 \\
\hline & fev & 0,00 & 0,13 & 2783 & 0,00 & 0,16 & 1317 \\
\hline
\end{tabular}


aproximadamente $26000 \mathrm{~m}$ ), indicando a influência de partículas de aerossóis e gases suspensos no ar antes do processo de remoção dos mesmos pela precipitação, sugerindo que foram emitidos tanto de fontes locais quanto regionais, como a queima de biomassa (queimadas) que ocorrem principalmente nos meses de agosto e setembro (PAULIQUEVIS et al., 2007). Já nos meses de dezembro e fevereiro os alcances diminuíram indicando a remoção dos compostos químicos atmosféricos pela precipitação, sugerindo que nestes meses as partículas e gases emitidos provieram de fontes locais urbanas.

Numa pesquisa que reforça a influência de emissões regionais nos meses de agosto e setembro em Cuiabá foram observadas, durante o período de 1992 e 1995, altas concentrações de material particulado inalável, com valores acima de $130 \mathrm{\mu g} \mathrm{m}^{-3}$ (ALMEIDA FILHO, 2006). No período chuvoso, predominam contribuições de poluição urbana, ou seja, emissões locais, as quais atingiram cerca de $13 \%$ da massa do aerossol no período chuvoso; entre outras como: $58 \%$ de emissões biogênicas, $28 \%$ de ressuspensão do solo e $1 \%$ de queima de biomassa (ALMEIDA FILHO, 2006).

$\mathrm{O}$ pH apresentou maior alcance que as demais variáveis, o que pode indicar a influência de outros compostos químicos alterando a acidez da precipitação, além dos ânions inorgânicos analisados, como por exemplo, íons orgânicos que não foram investigados neste trabalho, sendo que a acidez livre não explicada é normalmente atribuída aos ácidos orgânicos (LARA et al., 2001).

Os ânions $\mathrm{NO}_{3}^{-}$e $\mathrm{SO}_{4}^{2-}$ apresentaram semelhança em relação ao alcance em todas as avaliações, o que pode ser atribuída à co-emissão de seus precursores $\mathrm{SO}_{2}$ e $\mathrm{NO}_{x^{\prime}}$, assim como a semelhança de seus comportamentos químicos na precipitação (CAO et al., 2009). O coeficiente de correlação de Pearson confirmou esta forte correlação: 0,94 em outubro $(p<0,05), 0,64$ em dezembro $(p<0,05)$ e 0,87 em fevereiro $(p<0,05)$.

$\mathrm{O}$ alcance de dependência espacial é um parâmetro importante ao analisar os semivariogramas, ou seja, é a distância na qual as amostras estão correlacionadas espacialmente. Essa distância indica que vizinhos dentro da área com esse raio podem ser usados na estimativa de valores em espaçamento mais próximos (REICHERT et al., 2008).

Tanto os semivariogramas ajustados pelo método REML como OLS, 83\% apresentaram efeito pepita zero. $\mathrm{O}$ efeito pepita revela a descontinuidade do semivariograma em distâncias menores que as amostradas (VIEIRA, 2000), ou seja, está relacionado ao fato de que os dados não foram coletados a intervalos suficientemente pequenos para demonstrar o comportamento espacial da variável. Quando a distância não é suficiente para detectar o comportamento espacial ocorre o efeito pepita puro (BAÚ et al., 2006). O efeito pepita também pode estar relacionado a erros de medida ou pequenas variações não detectadas. Já neste estudo, os locais de amostragem foram suficientemente próximos para de- tectar a variabilidade espacial das variáveis químicas e fisíco-químicas da precipitação.

A variação do patamar pode ser explicada pela variabilidade temporal das variáveis estudadas, ou seja, a concentração dos elementos químicos em determinadas épocas do ano afetou a magnitude da variabilidade dos dados, caracterizada pelo patamar. O patamar de um semivariograma é o valor de variância correspondente ao ponto em que o mesmo estabiliza (ANDRIOTTI, 2009).

Os semivariogramas ajustados pelo método da máxima verossimilhança restrita estão apresentados na Figura 2.

\section{Conclusão}

O melhor método de ajuste dos semivariogramas foi o da máxima verossimilhança restrita, sendo recomendado para estudos associados à aplicação da geoestatística no mapeamento de variáveis químicas da precipitação pluvial.

A análise dos parâmetros dos semivariogramas auxiliou na compreensão das fontes de emissão de partículas atmosféricas que são removidas pela precipitação pluvial. Foi possível identificar em outubro, por meio do alcance, um raio de influência de partículas e gases suspensos no ar, removidos pela precipitação, que podem ter sido emitidos tanto de fontes locais como regionais, e em dezembro e fevereiro, com a diminuição dos valores de alcance, sugere-se que as fontes de emissão foram locais e urbanas.

\section{Agradecimentos}

Ao CNPq pela bolsa de estudos concedida.

\section{Referências Bibliográficas}

ALASTUEY, A.; QUEROL, X.; CHAVES, A.; RUIZ, C. R.; CARRALATA, A.; LOPEZ-SOLER, A. 1999. Bulk deposition in a rural área located around a large coalfired Power station, northeast Spain. Environmentl Pollution, 106, 359-367.

AL-KHASHMAN, O. A.; TARAWNEH, Q. Y. 2007.Spatial prediction model and its application to chemistry of atmospheric precipitation in Jordan. Atmospheric Research, 84, 399-409.

ALMEIDA FILHO, E. O. 2006. Avaliação das fontes de emissão de material particulado na atmosfera da cidade de Cuiabá. Dissertação (Mestrado), Instituto de Física, Universidade Federal do Mato Grosso, Mato Grosso.

ANDRÉ, F.; JONARD, M.; PONETTE, Q. 2007. Influence 
of meteorological factors and polluting environment on rain chemistry and wet deposition in a rural área near Chimay, Belgium. Atmospheric Environment, 41, 1426-1439.

ANDREAE, M. O.; ANDERSON, B. E.; BLAKE, D. R.; BRADSHAW, J. D.; COLLINS, J. E.; GREGORY, G. L.; SACHSE, G. W.; SHIPHAM, M. C. 1994. Influence of plumes from biomass on atmospheric chemistry over the equatorial and tropical South Atlantic during CITE 3. Journal of Geophysical Research, 99(D6), 12793-12808.

ANDREAE, M. O.; ARTAXO, P.; FISCHER, H.; FREITAS, S. R.; GRÉGOIRE, J. M.; HANSEL, A.; HOOR, P.; KORMANN, R.; KREJCI, R.; LANGE, L.; LELIEVELD, J.; LINDINGER, W.; LONGO, K.; PETERS, W.; REUS, M.; SCHEEREN, B.; SILVA DIAS, M. A. F.; STROM, J.; VAN VELTHOVEN, P. F. J.; WILLIAMS, J. 2001. Transport of biomass burning smoke to the upper troposphere by deep convection in the equatorial region. Geophysical Research Letters, 28(6), 951-954.

ANDRIOTTI, J. L. S. 2009. Fundamentos de Estatística e Geoestatística. $2^{\mathrm{a}}$ reimpressão. São Leopoldo: EDITORA UNISINOS, $165 \mathrm{p}$.

BAÚ, A. L.; GOMES, B. M.; QUEIROZ, M. M. F.; OPAZO, M. A. U.; SAMPAIO, S. C. 2006. Comportamento espacial da precipitação pluvial mensal provável da mesoregião oeste do Estado do Paraná. Irrigação, Botucatu, 11(2), 150-168.

BRAULE, R. 2001. Estatística aplicada com Excel: para cursos de administração e economia. Rio de Janeiro: Elsevier, $250 \mathrm{p}$.

BRASIL. Instituto Brasileiro de Geografia e Estatística - IBGE. Disponível em: <http://www.ibge.gov.br>. Acesso em: 08 set. 2012.

BURROUGH, P. A.; McDONNELL, R. A. 1998.Principles of geographival information systems. Oxford: Oxford University Press, 333p.

CAO, Y. Z.; WANG, S.; ZHANG, G.; LUO, J.; LU, S. 2009. Chemical characteristics of wet precipitation at an urban site of Guangzhou, South China. Atmospheric Research, 94, 462-469.

CARMO, C. N.; HACON, S.; LONGO, K. M.; FREITAS, S.; IGNOTTI, E., PONCE DE LEON, A.; ARTAXO, P. 2010. Associação entre material particulado de queimadas e doenças respiratórias na região sul da Amazônia brasileira. Revista Panam Salud Publica, 27(1), 10-16.
CRESSIE, N. 1993.Statistics for spatial data. New York: Wiley.

DIAS, V. R. M.; SANCHES, L.; ALVES, M. C; NOGUEIRA, J. S. 2012. Spatio-temporal variability of anions in wet precipitation of Cuiabá, Brazil.Atmospheric Research, 107, 9-19.

DURAND, P.;NEAL, C.; LELONG, F. 1992.Anthropogenic and natural contributions to the rainfall chemistry of a mountainous area in the Cevennes National Park (Mont-Lozere, southern France). Journal of Hydrology, 130, 71-85.

FLUES, M.; HAMA, P.; LEMES, M. J. L.; DANTAS, E. S. K.; FORNARO, A. 2002. Evaluation of rainwater acidity of the rural region due to a coal-fired Power plant in Brazil. Atmospheric Environment, 36(14), 2397-2404.

FONTENELE, A. P. G.; FEDROTTI, J. J.; FORNARO, A. 2009. Avaliação de metais traços e íons majoritários em águas de chuva na cidade de São Paulo. Química Nova, 32(4), 839-844.

FORNARO, A. 2006. Águas de chuva: conceitos e breve histórico. Há chuva ácida no Brasil? Revista USP, São Paulo, 70, 78-87.

GALPIN, J.; HELD, G. 2002.Analysis of rain quality data from the South African interior.Environmetrics, $13,333-346$.

HAN, G.; TANG, Y.; WU, Q.; TAN, Q. 2010.Chemical and strontium isotope characterization of rainwater in karst virgin forest, Southwest China. Atmospheric Environment, 44, 174-181.

KERRY, R.; OLIVER, M. A. Comparing sampling needs for variograms of soil properties computed by the method of moments and residual maximum likelihood. 2007. Geoderma, 140(4) 383-396.

LARA, L. B. L. S.; ARTAXO, P.; MARTINELLI, L. A.; VICTORIA, R. L.; CAMARGO, P. B.; KRUSCHE, A.; AYERS, G. P.; FERRAZ, E. S. B.; BALLESTER, M. V. 2001. Chemical composition of Rainwater and anthropogenic influences in the Piracicaba River Basin, Southeast Brazil. Atmospheric Environment, 35, 4937-4945.

LEAL, T. F. M.; FONTENELE, A. P. G.; PEDROTTI, J. J.; FORNARO, A. 2004. Composição Iônica Majoritária de Águas de Chuva no Centro da Cidade de São Paulo. Química Nova, 27(6), 855-861.

LOPHAVEN, S.; CARSTENSEN, J.; ROOTZÉN, H. 2004.Methods for estimating the semivariogram. In: 
LOPHAVEN, S. Design and analysis of environmental monitoring programs. Denmark: KongensLynby.

MAITELLI, G. T. 1994. Uma abordagem tridimensional de clima urbano em área tropical continental: o exemplo de Cuiabá-MT. Tese (Doutorado), Faculdade de Filosofia, Letras e Ciências Humanas, Universidade de São Paulo, São Paulo.

MATO GROSSO. Anuário Estatístico 2009. Secretaria de Estado de Planejamento e Coordenação Geral Superintendência de Estudos e Informações. Disponível em: <http://www.seplan.mt.gov.br/>. Acesso em: 09 jul. 2010.

MELLO, J. M.; BATISTA, J. L. F.; RIBEIRO JR., P. J.; OLIVEIRA, M. S. 2005. Ajuste e seleção de modelos espaciais de semivariograma visando a estimativa volumétrica de Eucalyptus grandis. ScientiaForestalis, 69, 25-37.

MIGLIAVACCA, D.; TEIXEIRA, E. C.; MACHADO, A. C. M.; PIRES, M. R. 2005. Composição Química da Precipitação Atmosférica no sul do Brasil - Estudo preliminar. Química Nova, 28(3), 371-379.

PAULIQUEVIS, T.; LARA, L. L.; ANTUNES, M. L. ARTAXO, P. 2007. Aerosol and precipitation chemistry in a remote site in Central Amazonia: the role of biogenic contribution. AtmosphericChemistryandPhysicsDiscussions, 7, 11465-11509.

OLIVEIRA, M. C. N. Métodos de parâmetros em modelos geoestatísticos com diferentes estruturas de covariância: uma aplicação ao teor de cálcio no solo. 2003. Tese (Doutorado), Universidade de São Paulo, São Paulo.

PIO, C. A.; SALGUEIRO, M. L.; NUNES, T. V. 1991. Seasonal and air-mass trajectory effects on Rainwater quality at the South-Western Europeas border. Atmospheric Environment, 25A (10), 2259-2266.

R Development Core Team. 2010. R: A language and environment for statistical computing. R Foundation for Statistical Computing, Vienna, Austria. URL: $<$ http://www.R-project.org>.

REICHERT, J. M.; DARIVA, T. A.; REINERT, D. J.; SILVA, V. R. 2008. Variabilidade espacial de Planossolo e procutividade de soja em várzea sistematizada: análise geoestatística e análise de regressão. Ciência Rural, 38(4), 981-988.

RIBEIRO JR., P. J.; DIGGLE, P. J. 2001. GeoR: A package for geostatistical analysis. R-NEWS, 1(2).
ROCHA, F. R.; SILVA, J. A. F.; LAGO, C. L.; FORNARO, A.; GUTZ, I. G. R. 2003. Wet deposition and related atmospheric chemistry in the São Paulo metropolis, Brazil: Part 1. Major inorganic ions in rainwater as evaluated by capillary electrophoresis with contactless conductivity detection. Atmospheric Environment, 37, 105-115.

RODRIGUES, R. A. R.; MELLO, W. Z.; SOUZA, P. A. 2007. Aporte atmosférico de amônio, nitrato e sulfato em área de floresta ombrófila densa Montana na Serra dos Órgãos, RJ. Química Nova, 30(8), 1842-1848.

SINGH, K. P.; SINGH, V. K.; MALIK, A.; SHARMA, N. MURTHY, R. C.; KUMAR, R. 2007. Hydrochemistry of wet Atmospheric precipitation over an urban area in Northern Indo-Gangetic Plains. Environmental Monitoring and Assessment, 131, 237-254.

SOUZA, P. A.; MELLO, W. Z.; MALDONADO, J. 2006. Composição química da chuva e aporte atmosférico na Ilha Grande, RJ. Química Nova, 29(3), 471-476.

TEIXEIRA, E. C.; MIGLIAVACCA, D.; PEREIRA FILHO, S.; MACHADO, A. C. M.; DALLAROSA, J. B. 2008. Study of wet precipitation and its chemical composition in South of Brazil. Anais da Academia Brasileira de Ciências, 80(2), 381-395.

TRANTER, M.; DAVIES, T. D.; ABRAHAMS, P. W.; BLACKWOOD, I.; BRIMBLECOMBE, P.; VINCENT, C. E. 1987. Spatial variability in the chemical composition of snowcover in a small, remote, Scottish catchement. Atmospheric Environment, 21, 853-862.

VIEIRA, S. R. 2000. Geoestatística em estudos de variabilidade espacial do solo. In: NOVAIS, R. F. et al. (Eds). Tópicos em ciência do solo. Viçosa: Sociedade Brasileira de Ciência do Solo, 1, 1-54. 